\title{
Power System Restoration Using Multilayer Perceptron
}

\author{
Deepak Kumar \\ Department of Electrical Engineering, Raja Balwant Singh Engineering Technical Campus, Agra, India \\ *Corresponding author E-mail: deepu1796@gmail.com
}

Manuscript received 1 Jan 2021; revised 10 Jan 2021; accepted 15 Jan 2021. Date of publication 20 Jan 2021

\begin{abstract}
In recent years, power systems are being operated nearer to their limits due to economic competition and deregulation. Also, nowadays the challenge is to include large and ever increasing amounts of decentralized generated power into the existing transmission network and at the same time comply with the electricity market transmission demands. Both factors increase the risk of blackout. After which, power needs to be restored as quickly and reliably as possible and, accordingly, detailed power system restoration plans are required. The multilayer perceptron network is chosen for a more precise examination.
\end{abstract}

Keywords: Power System Restoration; Artificial Neural Network; Multilayer Perceptron

\section{Introduction}

Power system major failures are very rare events. However, when outage occurs, the effects on commerce, industry, and general population life can be quite severe. After a blackout, a subject of critical importance is the rapidity with which electric service is restored. In order to minimize the economic and social costs of an outage, the majority of electric utility companies have pre-established norms and operating procedures to restore the power system. These guidelines and operating procedures contain steps that an operator should follow sequentially in order to restore the system. However, the highly stressful circumstances encountered after a blackout together with the fact that these guidelines are based on assumed the system conditions which may or may not be present, diminishes the success rate (defined as that fraction of restoration attempts which could not result in unacceptable loading and voltage profiles, or in breaker operations by the protection systems) of the technique.

The main reason for unsuccessful restoration attempts based on this technique is that the prevailing conditions of the power system can differ remarkably from the assumed conditions when the restoration plan was developed. How to quickly and orderly restore the electricity supply and reduce losses as could be possible from a system outage becomes a challenging issue for electrical engineers. The power system restoration can be divided into three sub-problems: unit start-up, network reconfiguration, and load recovery. Particularly, a comprehensive and effective network reconfiguration optimization scheme can accelerate the process of power system restoration, shorten restoration period, and reduce the possibility of equipment damages. The combinatorial analysis of proper restoration planning that involves a variety of restrictions and conditions, requires operators' judgment and comprehensive power system studies.

Restoration is an important part of Advanced Distribution Automation (ADA), which seeks to restore the un-faulted and out-aged parts of a system due to the isolation of a fault. Mathematically, the restoration problem is a combinatorial problem with the objective of maximizing the supply of power for as many customers as possible while satisfying source, line/cable loading, and often radial network constraints. Power system restoration is the procedure of restoring system elements such as generators, transmission lines, and load centers after a blackout. It is technically complex, time-consuming, and probably fail. Meanwhile, losses to the customers and to the industry mount rapidly. Prolongation of this process forces many economic and political costs to the system, which makes rapid as well as effective restoration very important. Power system restoration is a complex problem involving a large number of generation, transmission and distribution, and load constraints.

In, the restoration process is divided into three stages: preparation, system restoration, and system load restoration. Though, one common thread linking these stages is the generation availability at each stage. The restoration process involves finding suitable backup feeders and laterals to transfer the outage loads using operational criterion through a series switching operations. The criteria may differ from 
utilities to utilities. Some of them are the amount of restored load, the number of switching operations, and the degree of load balance. Selection of a restoration scheme is a process of looking for a combination of switching operations that satisfy multiple objectives having certain constraints. As such, distribution system restoration can be considered as a multi objective decision-making problem. Various restoration plans lead to different system configurations, which may affect the quality of electric utilities. Also, restoration tasks are normally performed in an emergency, so operators are time bound to find a suitable restoration plan. Therefore, a computerized information system that supports decision-making job is crucial to help operators assess quickly restoration plans during the restoration process.

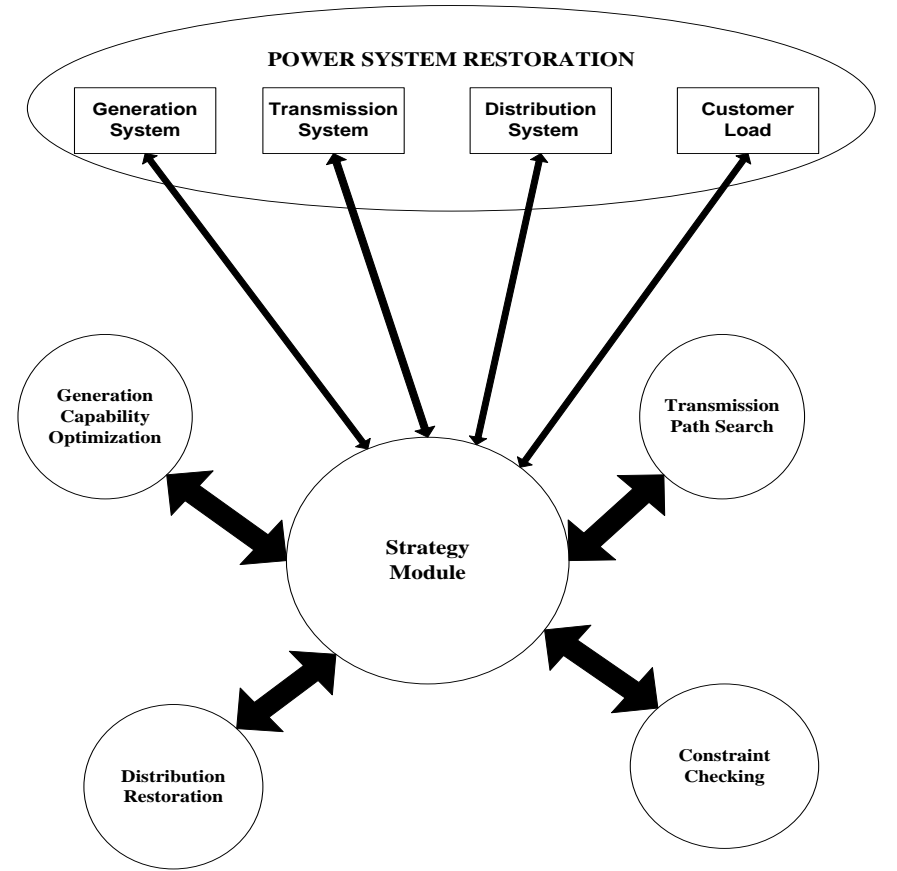

Fig.1. Power System Restoration Strategy

The overall goals of restoration should be to first rebuild a stable electric system, and then. Restore all remaining unserved load. In the process of attaining these goals, the following objectives need to be achieved as well:

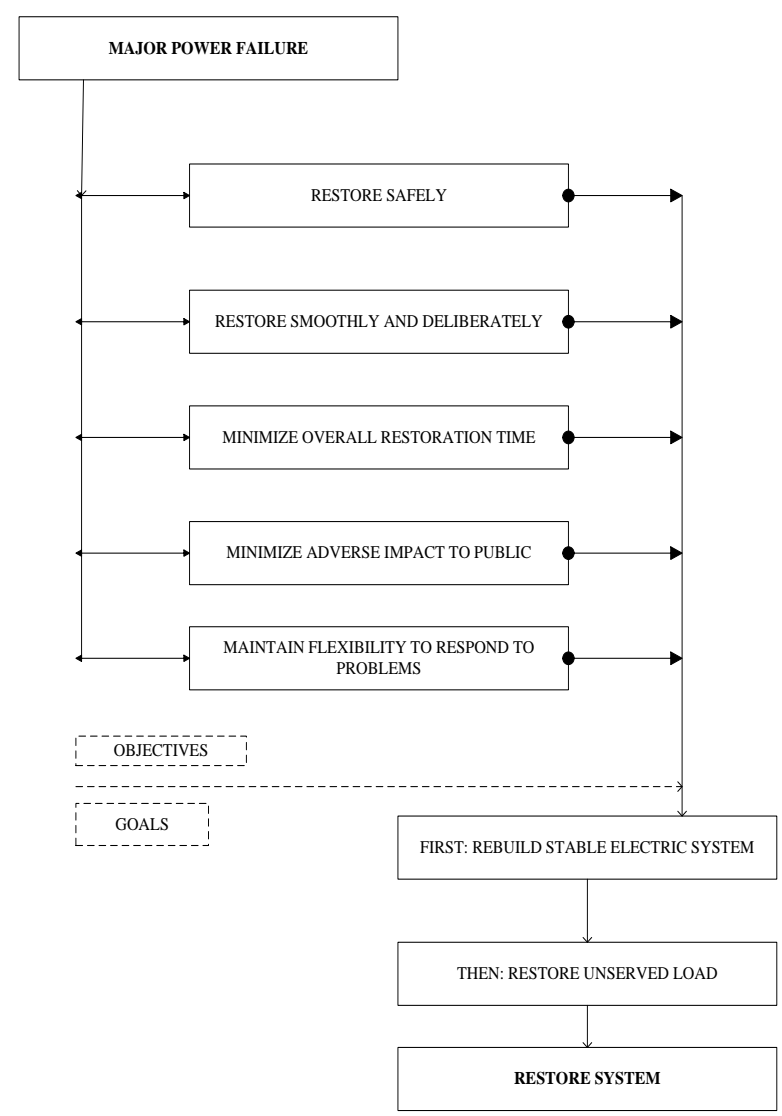

Fig.2. Flow Chart 


\section{Artificial Neural Network}

A neural network consists of interconnected elements called as neurons or nodes. The network is a parallel distributed process which solves a computational task. Artificial neural network functions are approximation and classification.

\subsection{Structure of the multilayer perceptron network}

The multilayer perceptron network is a universal network. This implies that a node cannot be separated to react only to certain parts of the given input vector. Mathematically, the multilayer perceptron network is capable of approximating any mathematical function.

\subsection{One-layer Perceptron}

The perceptron network is a non-linear mathematical model. It consists of nodes arranged in layers. Generally, all nodes in a layer are connected to all nodes in the adjacent layer with interconnecting weights. Weight matrix $\mathrm{W}$ and bias vector $\mathrm{b}$ determine the network output.

\subsection{Multilayer Perceptron}

The one-layer network is rarely used. The practical neural networks consist of two or more layers. They are called multilayer perceptron networks. Input layer is the first layer, then last output layer and all layers between them hidden layers. The information is given to the input layer which is propagated forward through the hidden layers to the output layer. Input layer connects each input node to each hidden layer node. All connections have a specific weight parameter. Nowadays interconnected power systems have become more and more complex. To prevent the insecure operation (major system failures and regional power blackouts) electric network utilities have installed SCADA systems to support computer based systems at the control centre. Computer systems contain applications for service quality, economic operation, operation control, system regulation and operational planning and scheduling. Before any security assessment or control action can be made, a system state must be estimated. The Matlab Neural Network Toolbox and the Neural Network Based System Identification Toolbox include functions for the back-propagation method. These use computer memory much more efficiently than Levenberg-Marquardt, but the convergence is very poor. The usability of these methods was found to be very limited in state estimation approach.

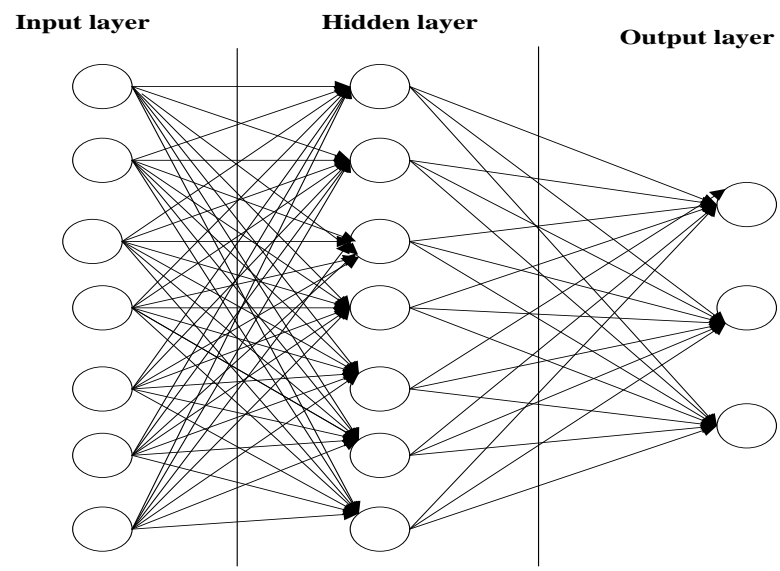

Fig.3. Neural Network Structure

\subsection{Perceptron learning algorithm}

- Dimensionality of the augmented input space

- Number of training patterns - size of the training epoch

- Generation of the training and validation sets

- Projection of the weight vector

- $\quad \mathrm{c} 0$ and $\mathrm{c} 1$ are vectors of pointers to input patterns belonging to the class 0 or 1

- The input patterns are plotted on the selected projection plane

- Patterns belonging to the class 0 , or 1 are marked with ' $\mathrm{o}$ ', or ' $\mathrm{x}$ ', respectively

- Superimposition of the projection of the separation plane which is a straight line

- Learning

- Initialisation of the weight vector with values from the range $[-1,+1]$

- Maximum number of training epochs

- The Perceptron Learning Law $\mathrm{cw}=\mathrm{cw}+1$

- The updated and normalised weight vector is stored in WW for feature plotting 
- $\quad$ projection of the weight vector

- After every pass through the set of training patterns the projection of current decision plane which is calculated with the help of current weight vector is plotted after the previous projection has been erased

\subsection{Training}

The neural network output will lead results similar to actual one, when it is properly trained. This is called generalization. This makes it possible to train a network with a set of input/output pairs and to get good results without training with all possible pairs. The number of hidden layers and nodes has to be selected carefully after several tests. If the local error minimum is not satisfactory, the number of nodes or layers should be increased. Large networks are not accurate in all cases due to over-fitting and convergence problems. Sometimes it is not possible to reduce the number of hidden layers and nodes due to accuracy, although the learning time is very long. In that case there are at least five possible solutions:

- Use Numerically Better Training Algorithm

- Divide The Problem Into Several Sub-Problems

- $\quad$ Reduce The Size Of Input Vector

- Use Pruned Network Structure Instead Of Fully Connected One

- Reformulate Problem

\subsection{Back-Propagation}

The training is generally based on output error minimization. The simplest training algorithm is the back-propagation method. The error is propagated backwards and the network parameters are updated in the direction of negative gradient with respect to the weight parameters (steepest descent gradient method). Unfortunately, the convergence of back-propagation is very slow. It requires a very small learning rate for stable learning.

\subsection{Reduction of training time}

The former case showed clearly the importance of the reduction of input nodes. In most cases the long training time is due to an overlarge neural network. The size of the neural network can be reduced with input node and connection reduction. However, there are only few sophisticated calculation methods for achieving this.

\subsection{Reduction of input nodes}

Reduction of input nodes would decrease the number of parameters. The reduction should be based on the knowledge from the identified system. This is very important, but also very difficult. One possible method is trial and error.

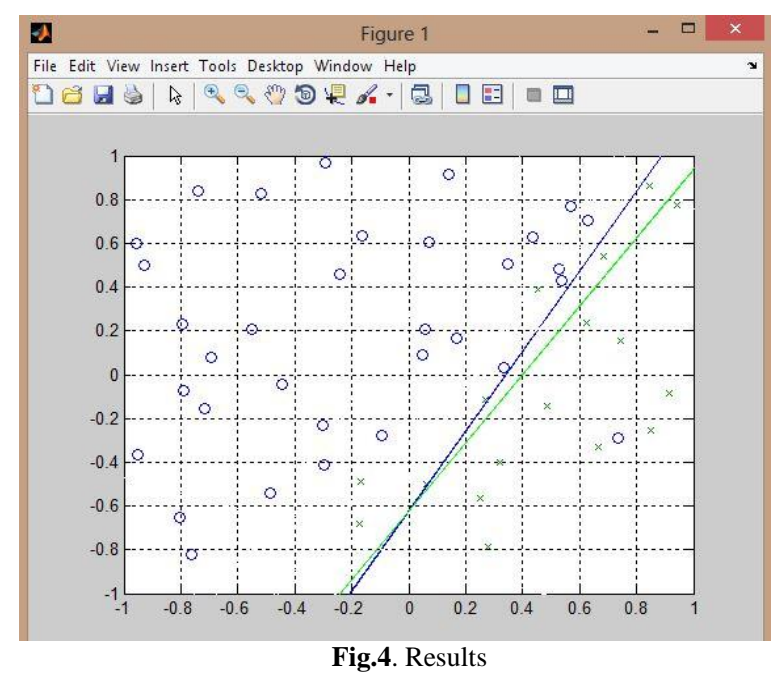

\section{Conclusion}

The neural network is a black box modeling tool. Its efficiency is based on its parallel computation capability. The neural network has the capability of modeling non-linear functions and its output can be calculated extremely fast. Mathematically it can be said that it is capable of modeling any function. The neural network can also be used as a classifier, for example in the decision-making process which can produce a final choice. Various applications based on neural networks have been reported in the power system area such as load forecasting, security assessment, protection of system, system control, fault diagnosis, alarm processing, system identification and operational planning. The most commonly used neural network has been a multilayer perceptron network. 


\section{References}

[1] Quiros-Tortos, J.; Wall, P.; Ding, L.; Terzija, V. Determination of sectionalizing strategies for parallel power system restoration: spectral clustering-based methodology. Electr. Power Syst. Res. 2014, 116, 381-390..

[2] LiBao Shi,"Determination of Weight Coefficient for Power System Restoration,"Ieee Transactions On Power Systems, Vol. 27, No. 2, May 2012.

[3] S.Nourizadeh et al."'Power system stability assessment during restoration based on a IET Gener. Transm. Distrib., Vol. 6,2012.

[4] S. Arash Nezam Sarmadi et al.,"A Sectionalizing Method in Power System Restoration Based on Wams,"Ieee Transactions On Smart Grid, Vol. 2, No. 1, March 2011.

[5] Cuong P. Nguyen et al.,"Agent Based Restoration With Distributed Energy Storage Support in Smart Grids,"Ieee Transactions On Smart Grid, Vol. 3, No. 2, June 2012.

[6] Wei Sun et al.,"Optimal Generator Start-Up Strategy for Bulk Power System Restoration,"Ieee Transactions On Power Systems, Vol. 26, No. 3, August 2011.

[7] Josz, C.; Fliscounakis, S.; Maeght, J.; Panciatici, P. AC Power Flow Data in MATPOWER and QCQP Format: ITesla, RTE Snapshots, and PEGASE. arXiv, 2016, arXiv:1603.01533.

[8] Fenghui Ren et al.,"Conceptual Design of A Multi-Agent System for Interconnected Automatic Power Systems Restoration,"Ieee Transactions On Power Systems, Vol. 27, No. 2, May 2012.

[9] El-Zonkoly, A.M. Renewable energy sources for complete optimal power system black-start restoration. IET Gener. Transm. Distrib. 2015, 9, 531-539. 